\title{
A propósito de teónimos indígenas en el conventus Emeritensis
}

\author{
J. V. Madruga Flores $Y$ J. Salas Martín*
}

\section{INTRODUCCIÓN}

En este trabajo de investigación ofrecemos una serie de inscripciones latinas votivas halladas en la provincia de Cáceres. Unas son inéditas, otras, por el contrario, están publicadas, aunque con lecturas e interpretaciones que no se ajustan a la realidad. Sus textos epigráficos hacen alusión a dedicaciones particulares a divinidades indígenas conocidas con anterioridad como Ataecina y Lacipaea o novedosa como Eci[r?]icu.

Especial relevancia adquiere Lacipaea, nombre que responde al parecer tanto a un teónimo como a un topónimo alusivo a la primera mansio de la vía terrestre que unía a Augusta Emerita con Complutum. El hallazgo de un ara consagrada a esta divinidad lusitana en la localidad cacereña de Conquista de la Sierra, nos ha permitido reinterpretar el texto epigráfico de otra inscripción votiva publicada con anterioridad y supuestamente dedicada a un dios indigena llamado Lac(ui), cuando en realidad corresponde a Lacipaea.

\section{INSCRIPCIÓN INÉDITA DE ATAECINA (Figura 1)}

Inscripción votiva inédita. Fragmento de ara de granito rojizo oscuro y granulado fino, de procedencia no local. Se halló durante el verano de 1992 formando parte del dintel izquierdo de una ventana semiderruida perteneciente a una vieja casa situada en la calle Cuesta, frente a un ancho corralón, muy cerca del conocido recinto fortificado denominado "Los Canchuelos", en la localidad cacereña de Salvatierra de Santiago. Actualmente permanece en el mismo lugar donde se encontró.

\footnotetext{
* Universidad de Extremadura.
} 


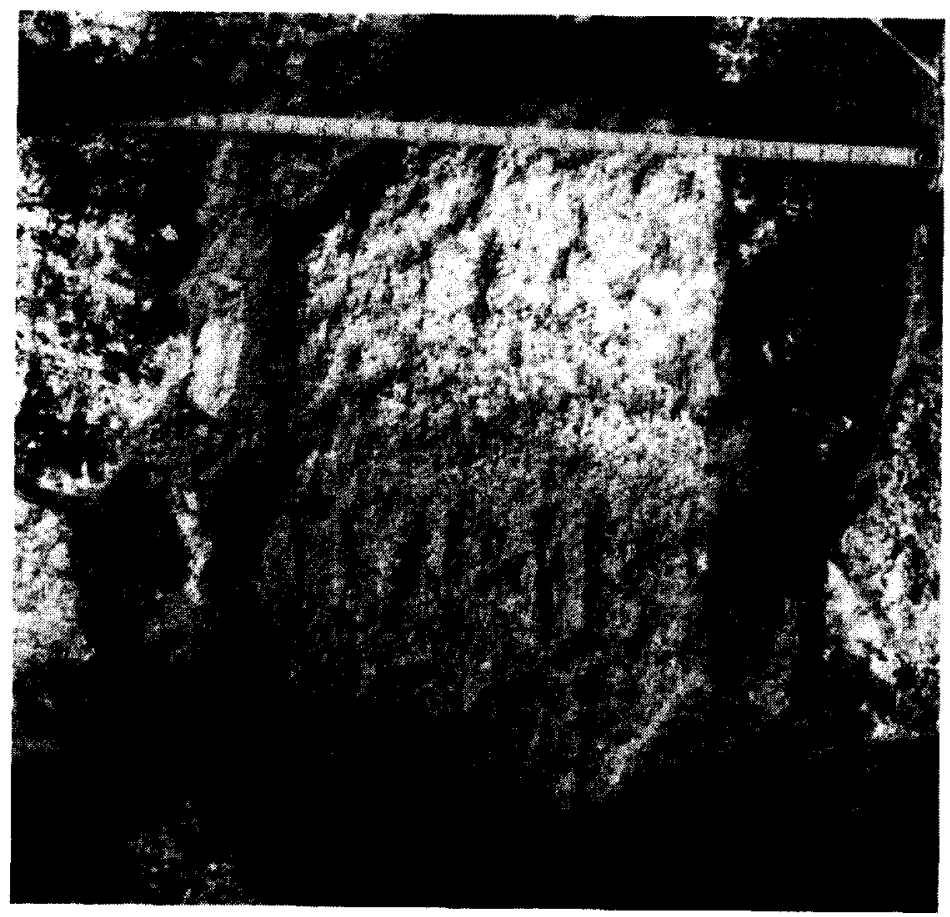

Figura 1

El epígrafe presenta una doble fractura que afecta a la parte central y a ambos laterales de su soporte material, y que ha hecho desaparecer parcialmente su texto epigráfico, del que se han conservado sólo parte del topónimo y del teónimo. Su actual ubicación no nos ha permitido comprobar la presencia en su coronamiento o bien de un foculus o tal vez, como suele ser habitual en otros epígrafes consagrado a Ataecina, de una o dos oquedades con formas cuadrangulares, rectangulares o irregulares para la fijación de uno o dos espigones. Tanto las dimensiones como las formas que adoptan estos huecos parecen descartar que se trate del tradicional foculus que suele acompañar a las aras votivas.

Nosotros creemos que estas oquedades sirvirian para la fijación de la representación iconográfica de una cabrita, animal vinculado al parecer a la diosa lusiana, en la parte superior de la cornisa del ara mediante uno o dos espigones. Esta sospecha la sustanciamos en el conocido epígrafe de Malpartida de Cáceres dedicado a Ataecina, donde aparece una inscripción grabada en una pequeña placa de bronce adosada a las patas de 
una cabrita, del mismo material, y que frontalmente mira en dirección al texto epigráfico '.

Las dimensiones visibles del monumento son de $30 \mathrm{cms}$. de largo, por $18 \mathrm{cms}$. de ancho. La altura de las letras oscila entre $5-4,5 \mathrm{cms}$. en Línea 1 y entre 4,5-4 $\mathrm{n} \mathrm{cms}$. en Línea 2, 10 que hace que el espacio interlineal varie de $2-1,5 \mathrm{cms}$. Las letras, muy desgastadas, dada la mala calidad del granito, son capitales cuadradas con rasgos rústicos.

La lectura del campo epigráfico del ara dice lo siguiente:

$$
\begin{aligned}
& \text { [-circ.-2-3] TVRIBR [-circ.-1-2] } \\
& \text { [-circ.-2-3] AEGIN [-circ.-2-] }
\end{aligned}
$$

Transcripción: [D(eae) D(ominae) o D(eae) D(ominae) S(anctae)?] TVRIBR[i(gensi)?] / [-Ad?]AEGIN[-ae]

Comentario: En la Línea 1, a la letra «T» le falta la mitad izquierda de su trazo horizontal. El arco de la primera " $R$ " no está cerrado. La segunda "R" aparece sólo con el rasgo vertical y la parte inferior del arco. En la Lín. 2, la "A" aparece con la mitad del rasgo inclinado izquierdo y sin la delineación horizontal. La "l" está grabada con remates muy marcados y de la "N" sólo se aprecia el ángulo superior izquierdo.

Qué duda cabe que las fracturas que presenta el ara en ambos laterales afecta a algunas de las letras del campo epigráfico conservado, planteando algún que otro problema su reconstrucción. En la Línea 1, concretamente en su margen izquierdo, cabe la posibilidad de la ausencia de 2 o 3 letras. Esta sospecha la sustanciamos en el número de grafías que faltan para configurar presumiblemente el nombre completo de la diosa lusitana en la Línea 2 del texto epigráfico, y que debe responder, siempre que nos fijemos en los paralelos que ofrecen las inscripciones halladas en la vecina localidad de Alcuescar 2, a Adaecina, Adegina, Attegina, Addaegina, Eadegina.

\footnotetext{
1 Vid. CIL II, 5298; FITA, F. “Inscripciones romanas inéditas de Cáceres, Brandomil y Lérida" $B R A H V I, 1885$, pp. 430-432.

2 Caballero Zoreda, L. y Rosco Madruga, J.: «Iglesia visigoda de Santa Lucia del Trampal, Alcuéscar (Provincia de Cáceres). Primera Campaña de Trabajos Arqueológicos. 1983-4. "Extremadura Arqueológica 1, 1988, p. 248, n. ${ }^{\circ}$ 1. Y también Caballero Zoreda, L.; Almagro Gorbea, A.; Madroñero de la Cal, A.; Granda Sana, A.: "La Iglesia de época visigoda de "Santa Lucía del Trampal. Alcuéscar (Cáceres)" Extremadura Arqueológica II, 1991, pp. 508, cuadro 3. Y un trabajo más reciente de SALAS MARTín, J. y RoSCO MADRUGA, J. "Epigrafia latina votiva de Santa Lucia del Trampal I (Alcuéscar, Cáceres)". Norba 14, 1995, (en prensa).
} 
Si esta conjetura fuese cierta, es probable que el teónimo estuviese acompañado, eso sí, de forma abreviada, de los títulos $D($ eae $)$ - S(anctae) • $D$ (ominae) o simplemente de $D(e a e) \cdot D o m i n a e$. En todo caso, es una posibilidad que no conviene rechazar de antemano.

El hallazgo de un epígrafe votivo consagrado a Ataecina no es novedoso ni aíslado en Salvatierra de Santiago, pues ya en el siglo pasado se exhumó otro ara en esta localidad, y aunque ha pasado desapercibida a los investigadores también está dedicada a la diosa lusitana (Vid. inscripción $n . \stackrel{0}{2}$ ).

Ataecina es una deidad indígena, al parecer de carácter infernal, cuyo culto continuó bajo la dominación romana, asociándose a través del conocido proceso de interpretatio Romana a Proserpina. Al menos esto es lo que se desprende tanto de la conocida inscripción conteniendo una tabella defixioni a Ataecina Proserpina, hallada en el pantano romano llamado hoy de Proserpina y con anterioridad de la Albuera ${ }^{3}$, como de otro epígrafe encontrado en la calle Cardenas, de Mérida, cuyas abreviaturas parecen que también corresponden a Ataecina Proserpina ${ }^{4}$.

Afortunadamente, los hallazgos de inscripciones consagradas a Ataecina se han multiplicado en los últimos años y hoy en día superan con amplitud la treintena de testimonios, gracias, sobre todo, a la reciente aportación de un importante número de epígrafes exhumados en la iglesia visigótica de Santa Lucía y zona del Trampal, en Alcuéscar (Cáceres) ${ }^{5}$.

El ámbito de difusión del culto a Ataecina se concentra básicamente en la Extremadura centro-occidental, zona Alentejo y parte occidental de Toledo, territorios que pertenecían en época romana a la provincia Ulterior Lusitania.

En otro orden de cosas, queremos resaltar que la pequeña localidad cacereña de Salvatierra de Santiago, cuyos territorios pertenecieron antaño al ager coloniae de Norba Caesarina, ha sido siempre generosa en la aportación de inscripciones latinas. Y buena muestra de ello es que actualmente nos ofrece unos veinticinco epígrafes, destacando aquellos que contienen teónimos de claro sabor lusitano.

3 CIL II, 462; J. Álvarez Saenz de Buruaga, J.M.: «El hallazgo de la lápida de Proserpina de Mérida" Revista Estudios Extremeños. II, 1955, pp. 325-401. Su texto dice lo siguiente: Dea Ataecina Turi/brig(ensis) Proserpina / pertuam maiestatem / te rogo oro obscro / uti vindices quot mihi / furti factum est quisquis / mihi imudavit / involavit / minvusve fecit [e]a[s res] q(uae) i(nfra) $\mathrm{s}$ (criptae) s(unt) tunicas vi [--] [pa]enula / lintea ii in[dus]ium cu/ivs [--- $\mathrm{m}$ ignoro/---.

4 El epígrafe se encuentra actualmente perdido. Su texto, según el CIL II 461, decia lo siguiente: $\mathrm{D}$ (eae) S(anctae) A(taecinae) T(uribrigensi) P(roserpinae).

5 Vid. cita n. ${ }^{\circ} 2$. 
Roso de Luna fue el primer autor que dio a conocer aras votivas halladas en este pueblo cacereño, concretamente dos, una dedicada a Júpiter, acompañado de un epíteto que plantea serios problemas de lectura ${ }^{6}$; y otra, a Ataecina (Vid. Inscripción n.o 2 del trabajo). Se documentan en esta localidad, además, otros epigrafes votivos consagrados a Mercurio Colu, en este caso como dios romano indigenizado del comercio y artesanos ${ }^{7}$; a la deidad lusitana Aerbina ${ }^{8}$, que durante mucho tiempo se consideró como testimonio único ${ }^{9}$ y cuya función religiosa permanece aún desconocida. Recientemente se ha encontrado un epígrafe dedicado a la diosa romana Victoria ${ }^{10}$.

\section{INSCRIPCIÓN DE ATAECINA (CORRIGENDA)}

En el año 1904 Roso de Luna publicó un artículo sobre epigrafía cacereña donde daba a conocer, entre otros epigrafes latinos, el texto in-

- Roso dE LUNA, M. "Nuevas inscripciones romanas de la región norbense" $B R A H$ XLIV, 1904, p. $122, n^{\circ} 8$, realizó la siguiente lectura: lovi De(o) / Eso P(osuit). Últimamente, ARMIN U. Stylow (en $H E p 4,1994,247$ ) propone $D e / f e(n)$ sor[i], atributo hasta ahora desconocido, pero que es comparable a Depulsori, que ya está constatado.

7 Mercu/rio Colu/ali Q(uintus) N(orbanus) / Saturnin(us) / a(nimo) l(ibens) v(otum) s(olvit) (Vid. Roso de LunA, M. “Nuevas inscripciones, op. cit. p. $121 \mathrm{~m} \mathrm{n.}{ }^{\circ} 5$; Idem. en Revista de Extremadura VIII, Cáceres 1906, p. 121, n. ${ }^{\circ}$; RAMÓn MÉlIDA, J.R. Catálogo Monumental de España. Provincia de Cáceres (1914-1916). Madrid 1924, p. 196, n. ${ }^{\circ} 457$; HuRTAdo dE SAN ANTONIO, R. Corpus Provincial de Inscripciones Latinas (Cáceres). (=CPILC), Cáceres 1977, n. 426). Tradicionalmente se ha considerado que el epiteto de Mercurio quizá tenga que ver con el indoeuropeo "kuel"girar" (Vid. Albertos Firmat, M.L. La onomástica personal primitiva de Hispania. Tarraconense y Bética. Salamanca, 1965, p. 93) y por ello se ha interpretado como protector de las labores caseras. Sin embargo, considerando esa misma caracteristica filológica y el carácter de dios de la economía que Mercurio tiene, sería mejor interpretar el epíteto como dios protector de los carreteros, es decir, dios de aquellos que se dedicaban al transporte de mercancias. Refuerza esta interpretación el hecho de haber encontrado otra dedicación a Colvau en el Puente de la Macarrona en El Batán (Cáceres), junto a un ramal viario que uniria Caurium con la Vía de la Plata (Vid. Salas Martín, J. "El dios indigena Colu o Culuos. Aportaciones epigráticas". Veleia, II, 1987, p. 127 nota 1).

a Vid. Ramón y FeRnÁndez OXEA, J. "De epigrafía cacereña" BRAH CXXVIII, 1951, pp. 174175, n. 7 foto y CPILC, 438: Aerbin(ae) C(aius) Sem/proni/us Avitu/s l(ibens) a(nimo) v(otum) $s$ (olvit). La naturaleza femenina del teónimo no ha estado claro hasta que en 1988 se publicaron dos aras de Idanha a Nova (Portugal) dedicadas a Erbine. (HEp 4, 1994, 1042 y 1043). A pesar de ello, hay que señalar que en las fichas de M. L. ALBERTós Firmat, que se conservan en el Fichero General Epigráfico de Hispania de la Universidad Complutense, figura esta frase refiriéndose a la L.1 del epigrafe: "el final casi parece $M$ pero no es seguro". ¿Intuyó un nexo NA? Es muy probable, pero hemos querido confirmarlo y el ara ha desaparecido.

9 Vid. la nota anterior.

10 Vid. GonzÁlez CORdero, A., et alii "Nuevas aportaciones a la epigratía de Extremadura" Alcantara XXI, 1990 , pp. 139- 140 , n. ${ }^{\circ} 19$ foto y dibujo. Y en $H E p 2,1990,221$. Su texto dice lo siguiente: Victor/iae C(aius) lu/l(ius) Iulian/us a(nimo) I(ibens) / v(otum) s(olvit). 
completo de un ara procedente de la localidad cacereña de Salvatierra de Santiago, resaltando que «A pesar de su excelente conservación, resulta ilegible, gracias a la torpeza, tan frecuente, de haberla cubierto en su costado derecho por el arco de una escalera recientemente construída. Parece ser un ara consagrada a Ataecina, a juzgar por el $5 . .^{\circ}$ y 8. . renglón" ".

Según su editor, decía lo siguiente:

$\begin{array}{cl} & \text { DO... } \\ & \text { NA... } \\ & \text { VRII... } \\ & \text { ADD... } \\ 5 \quad & \text { INA... } \\ & \text { IVL. } \\ & \text { ADI... } \\ & \text { VS... }\end{array}$

Es verdad que Roso de Luna no reconstruyó el texto del epígrafe, pero intuyó con clarividencia que se trataba de un ara erigida en honor a Ataecina. Años después, Mélida recogía de nuevo el texto de este epígrafe en su conocido Catálogo Monumental de la Provincia de Cáceres, apuntando la ilegibilidad del epígrafe por "estar tapada por el arco de una escalera en casa de Jerónima Tejada" ${ }^{12}$. Este autor también resaltaba en su trabajo, siguiendo la información aportada con anterioridad por Roso de Luna, que al parecer se trataba de un ara consagrada a Ataecina, pero no entraba en su trabajo en más valoraciones personales acerca del contenido de la inscripción.

En 1977, Hurtado de San Antonio ${ }^{13}$ recoge el texto epigráfico del ara publicado con anterioridad por Roso de Luna y Mélida en su conocido Corpus Provincial de Inscripciones Latinas de la Provincia de Cáceres, calificando su contenido de naturaleza votiva, pero no se adentra en cuestiones relacionadas con la transcripción e interpretación del epigrafe.

11 Roso de Luna, M. "Nuevas inscripciones, op. cit. p. 123, n. 10.

12 Ramón MÉlIDA, J.R. Catálogo, op. cit, p. 196, n. 462.

13 CPILC, 432. 
Así pues, Roso de Luna fue el único autor que, al parecer, tuvo la ocasión de ver y leer personalmente el epígrafe de Salvatierra de Santiago, aunque tampoco descartamos la posibilidad de que algún erudito local ejerciese como corresponsal y cediese a este autor toda la información sobre el ara para su posterior publicación.

A primera vista, el texto de la inscripción, y siempre y cuando la lectura ofrecida por su editor sea fiable, hacía alusión a Ataecina, acompañada en este caso del título de Domina y del topónimo abreviado Turib(rigensis o rigae?).

Esta sospecha generó en nosotros el deseo de intentar localizar el epigrafe en Salvatierra de Santiago. Sin embargo, los más de noventa años transcurridos desde su publicación han representado un listón insuperable de recuperarlo. No obstante, no es descartable la posibilidad que en un futuro inmediato, como ha ocurrido otras veces con otras inscripciones, se "redescubra" el epígrafe y tengamos la ocasión de estudiar su texto personalmente.

El contenido del ara aparentemente no ofrece excesivas dificultades de interpretación en tanto en cuanto es el usual en las inscripciones votivas de la zona y en particular de Ataecina. La lectura y transcripción que seguidamente proponemos del texto epigráfico del ara es la siguiente:

$\begin{array}{ll} & \text { DO[-circ.-2]- } \\ & \text { NA[circ.-2 ]- } \\ & \text { VRIB[circ.- 2] } \\ & \text { ADD[circ.-3]- } \\ 5 \quad & \text { INA[circ. - 1] } \\ & \text { IVL } \cdot[\text { circ.-1]- } \\ & \text { ADI[circ.- 2] } \\ & \text { V(otum) S(olvit) [circ.- 2] }\end{array}$

Transcripción: DO[mi]/NA[e T]/VRIB[ri(gensi)] ADD[aeg?]/INA[e] / IVL(ius) • [B]/ADI[us]/ V(otum) S(olvit) [L(ibens) A(nimo)]

Comentario: Las dificultades que se aprecian en el texto del epígrafe editado por Roso de Luna aparentemente no son dificiles de superar. En Linea 1-2 no hay inconveniente en añadir las tres o cuatro letras que, creemos, faltan: Do[mi]/na [T] o bien Do[mi]/na[eT]. 
La Línea.3 es, si cabe, fácil de restituir: la última supuesta «|» correspondería al trazo vertical de la «B» que no fue tapado por la obra del arco de la escalera (sic) "urib».

Es posible también que le faltara alguna letra más y entonces tendríamos "urib[r]» o "urib[ri]» por el poco espacio que ocupan las «|». En las Líneas. 4-5 nos encontramos "Add...ina»., que, completada, nos daría: Add[aeg]/ina[e], con «ae» en la segunda sílaba del teónimo, tal y como se presenta en el epígrafe anterior. En las Lineas. 6-7 encontramos el nomen del dedicante y a partir del punto el inicio del cognomen, que seguiría en Lín. 7 y del que quedan algunas letras. $Y$ en Lín. 8 encontramos o bien la terminación en "vs" de cualquier nombre o bien el inicio de la fórmula votiva.

La restitución del cognomen del dedicante aparentemente es correcta habida cuenta de la atestiguación de un tal M(arcus) lulius Badius en la cercana localidad de Robledillo de Trujillo ${ }^{14}$, como oferente de otro epígrafe votivo, en este caso dedicada a la diosa romana Salus.

\section{INSCRIPCIÓN AL DIOS ECI [r?]ICV (CORRIGENDA) (Figura 2)}

Ara votiva de granito con zócalo, fuste con bandas horizontales a modo de moldura y cuyo coronamiento está dañado. Se encontró hace bastantes años en la finca denominada "Nava del Rey", situada en el término municipal de la localidad cacereña de Villamiel. Actualmente está depositada en el Museo Arqueológico Provincial de Cáceres.

Las dimensiones del monumento son de $65 \mathrm{cms}$. de largo, por $35 \mathrm{cms}$. de ancho, por $18 \mathrm{cms}$. de grosor. La altura media de las letras oscila entre 5 y $6 \mathrm{cms}$. Las letras son capitales cuadradas. $Y$ los signos de interpunción son circulares.

En 1899 Daniel Berjano ${ }^{15}$, correspondiente de la Academia de la Historia, dio a conocer el dibujo de una inscripción donde recogía su texto epigráfico de una forma incompleta y errónea, resaltando que se trataba de un ara consagrado a una supuesta diosa nominada Egina, nombre que haría alusión, consecuentemente, a Ataecina.

Al año siguiente de la publicación del epígrafe, el Marqués de Monsalud ofrece, gracias a una copia cedida por Daniel Berjano, una nueva lectura e

14 HEp 3, 1993, 135: Saluti /Marcus / lulius Ba/dius vot/um a(nimo) I(ibens) s(olvit).

15 Berjano EscobaR, D. “Nota Epigráfica" Revista de Extremadura I, 1899, pp. 121-122, n. 4. 


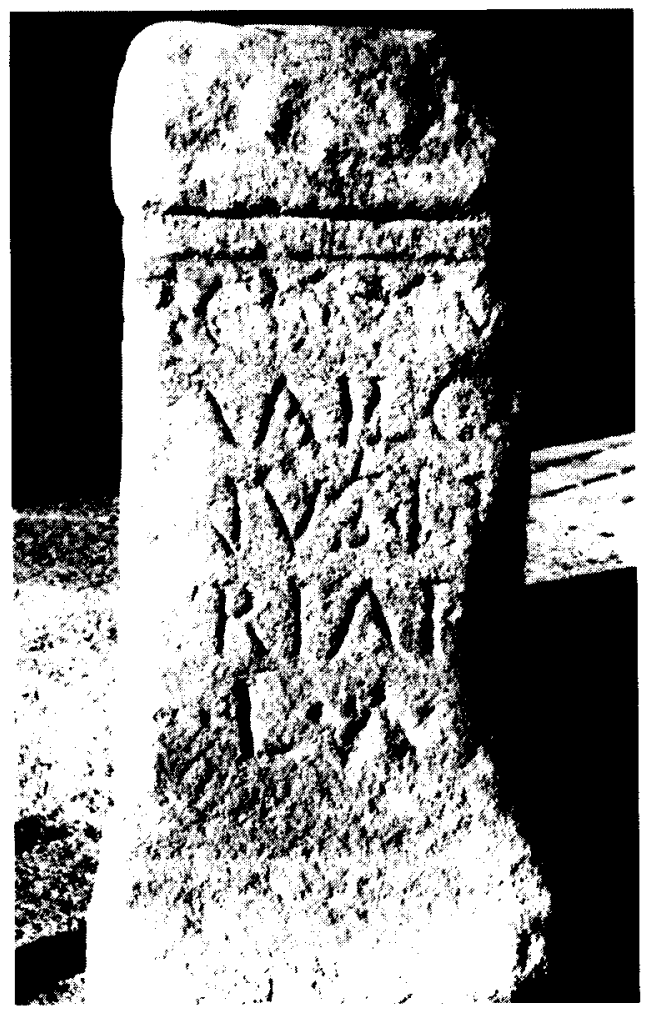

Figura 2

interpretación de su texto, apuntando que el ara estaba dedicada a unos supuestos Laribus Turibrigensibus ${ }^{16}$ :

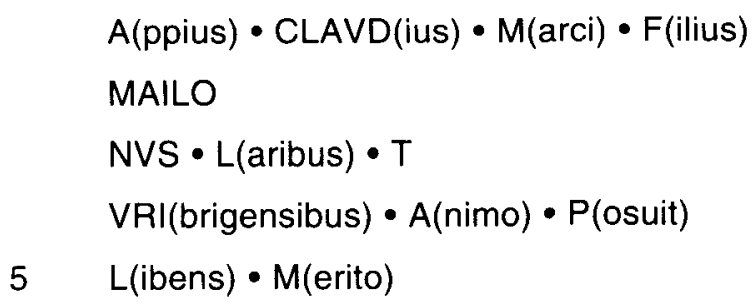

16 MARQUÉS DE MONSALUD “Nuevas Inscripciones romanas de Extremadura" BRAH XXVII, 1900, pp. 322-323, n. ${ }^{\circ} 1$. 
La interpretación del ara ofrecida por el Marqués de Monsalud no satisfizo en absoluto a Hübner ${ }^{17}$, quién a través de un calco que le remitió Juan Sanguino ofrece una nueva lectura de su texto epigráfico, no atreviéndose, sin embargo, a reconstruirlo:

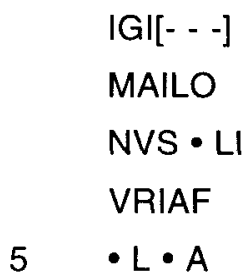

Mallón y Marín ${ }^{18}$ también ofrecieron una nueva lectura del texto del ara con motivo de la revisión de la obra epigráfica del Marqués de Monsalud:

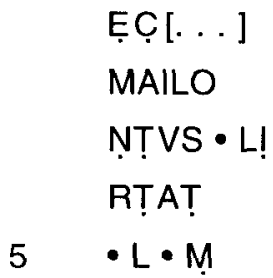

Con posterioridad, Callejo Serrano ${ }^{19}$ propuso una nueva lectura ara votiva de Villamiel:

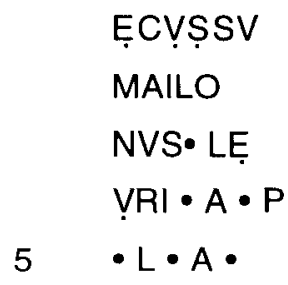

17 EE IX, 125.

18 Mallón, J. y Marín, T. Las inscripciones publicadas por el Marqués de Monsalud 1897. 1908. Estudio Crítico. Madrid 1951, p. 91, n. 185.

19 Callejo Serrano, C. "Aportación a la epigrafía del Campo Norbense" BRAH CLVII, 1965 , pp. 74-75, Lám. XXXIV. Esta lectura también es seguida por MELENA, J.L. “Jálama y la epigrafía del antiguo Corregimiento" Symbolae Ludovico Mitxelena Setuagenario Obaltae. Pars Prior. Vitoria 1985, p. 489; Idem. HEp 1, 1989, 206. 
Las discrepancias existentes entre los distintos autores a la hora de ofrecer la lectura e interpretación de este ara ha sido el revulsivo que nos ha impulsado a realizar una autopsia de su neto inscrito. Gracias a ella, hemos podido comprobar que de todas las lecturas ofrecidas hasta el momento, la de Callejo Serrano es la más ajustada a la realidad, excepto en la primera línea. La lectura e interpretación que proponemos de su texto epigráfico es la siguiente:

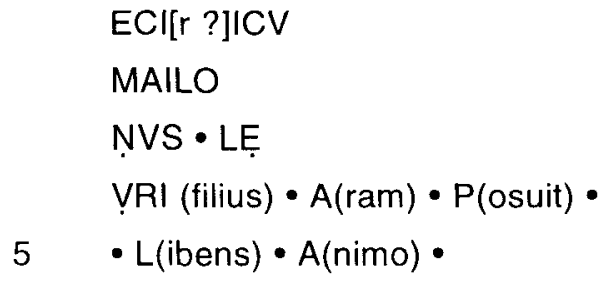

El soporte material del ara está dañado en ambos laterales, afectando a alguna de las letras de su neto inscrito. Ahora bien, la mayor dificultad de lectura del campo epigráfico la ofrece, sin lugar a duda, la Línea 1, que hace alusión al teónimo, ya que los trazos de algunas de sus grafías están muy desgastados y otras han desaparecido.

Para nosotros, el epigrafe no está consagrado, como pretendía Callejo Serrano, a un supuesto dios indígena nominado Ecussu, sino a una deidad desconocida llamada Ecilr ?]icu. De la letra desaparecida del nombre del teónimo, se aprecian ligeros trazos que invitan a creer que estamos frente a una posible "R», grafía que configuraría el teónimo Ecilrlicu. Pero esta posibilidad no es segura.

En cuanto al dedicante del ara, el sistema onomástico que lleva (nombre único + filiación patronímica) denuncia que estamos frente a un peregrinus. Mailonus y Leuri (Genitivo) son antropónimos de raigambre indigena que están representados en la epigrafía de la actual provincia de Cáceres ${ }^{20}$.

\section{INSCRIPCIÓN INÉDITA DE LACIPAEA (Figuras 3 y 4)}

El ara fue encontrada a finales de 1992 por Don M. Ciudad Fernández en la finca denominada indistintamente "Suerte de Portera" 0 "Los

20 CPILC, pp. 368 y 369. 


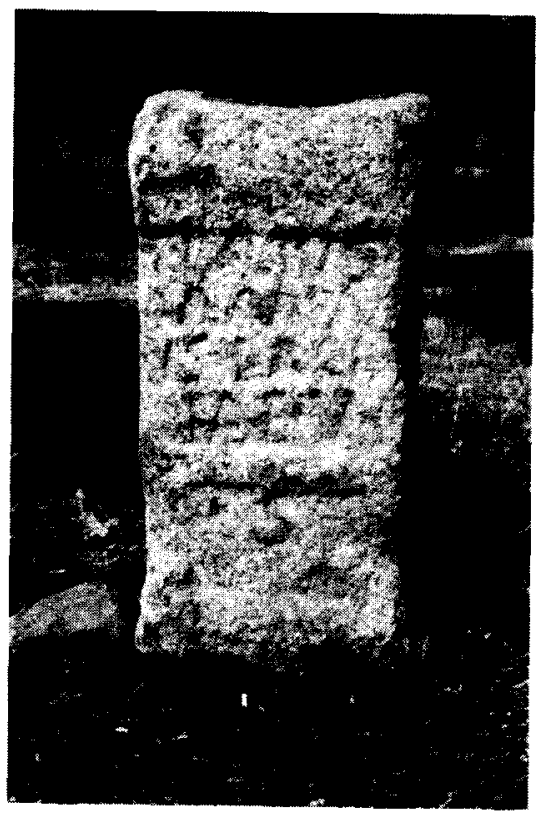

Figura 3

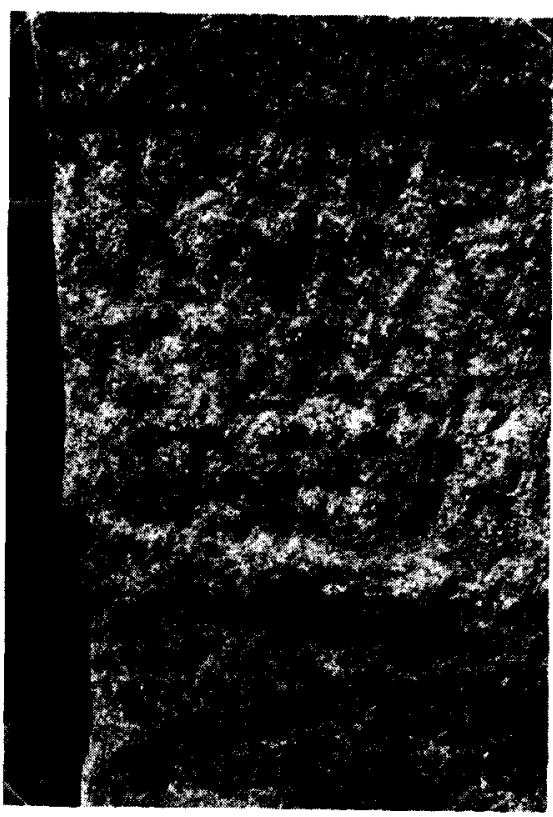

Figura 4 
Bonales", situada en el término municipal de Conquista de la Sierra (territorios pertenecientes antaño casi con seguridad a la Praefectura Turgaliensis, dependiente administrativamente de Augusta Emerita). Formaba parte de una pared semiderruída. Actualmente adorna los accesos a la casa que se construyó en su propiedad su descubridor, situada próxima a la ermita de Portera, en la localidad cacereña citada.

El ara de granito rosáceo muy bien labrada en sus cuatro caras presenta un zócalo con plinto moldurado, el cuerpo central, en el que hallamos un bocelete de $5,5 \mathrm{cms}$. que lo divide y que sirve de separación del tercio inferior, y el coronamiento, formado por una cornisa moldurada y por el cimacio, en el que se aprecia el foculus, que es de forma elíptica y tiene dos realzados longitudinales, paralelos al diámetro mayor, que sobresalen y lo atraviesan, dividiéndolo en tres pequeños receptáculos.

El estado de conservación del soporte es en general bueno, aunque presenta algún que otro desgaste en ambos laterales que afecta levemente al texto epigráfico.

Las dimensiones del monumento son de $54 \mathrm{cms}$. de largo, por $22 \mathrm{cms}$. de ancho, y $20 \mathrm{cms}$. de grosor. La altura de las letras oscilan entre 5 y 4 cms. y están muy desgastadas. Son capitales cuadradas con rasgos rústicos. La interpunción que presenta es redonda.

La lectura y transcripción del texto del epigrafe es la siguiente:

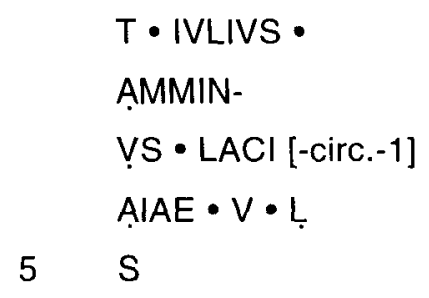

Nexos: Línea 2 AM

Transcripción: T(itus) • IVLIVS • /AMMIN/ṾS • LACI[p o b ?] /AIAE $V$ (otum) $\bullet L$ (ibens)/ S(olvit)

El soporte material del ara presenta ligeros desconchamiento en ambos laterales que afectan a algunas de las letras de su texto epigráfico, muy desgastado actualmente por la mala calidad del granito. A pesar de ello, su neto inscrito se puede leer aparentemente en su integridad.

Las mayores dificultades de lectura las ofrecen las Líneas 3 y 4 , precisamente aquéllas que hacen alusión al teónimo del epígrafe. En la primera 
de ellas se aprecían con claridad las letras "Laci». Trás el último carácter expuesto, es decir, la consonante «l», observamos un pequeño rasgo vertical que, por el contexto, creemos que corresponde al trazo vertical de una " $P$ » $O B$ ", pues hay espacio suficiente para que el lapicida grabe una letra. En la Línea 4, también su primera grafía está muy deteriorada, observándose restos de una "A".

Parece claro que el nombre del teónimo del ara corresponde a una divinidad lusitana conocida con anterioridad en la epigrafía latina del conventus Emeritensis bajo las formas Lacipaea o Lacibaea, y cuyos testimonios estaban atestiguados con anterioridad exclusivamente en Mérida, Albalá y también, como veremos más abajo, en Torre de Santa María.

El dedicante, T. lulius Amminus lleva un gentilicio muy frecuente en esta parte de Hispania ${ }^{21}$. Su cognomen, en cambio, está poco documentado en la epigrafía peninsular. Sus hallazgos se atestiguan, entre otros lugares, en Idanha la Velha (la antigua Civitas Igaeditanorum) ${ }^{22}$, Vila Viçosa ${ }^{23}$, Belver ${ }^{24}$ y en la localidad cacereña de Santa Marta de Magasca ${ }^{25}$. Parece claro, además, que Amminus es un atropónimo de raigambre celta ${ }^{26}$. $T$. Iulius Amminus está en posesión de la ciudadanía romana, aunque su cognomen denuncia un origen hispano.

\section{INSCRIPCIÓN DE LACIPAEA (CORRIGENDA)}

Roso de Luna publicó hace bastantes años un ara latina consagrada a una divinidad cuyo teónimo aparecía abreviado, $L A C$ (sic), en el texto del epígrafe. Fue encontrada en Torre de Santa María (Cáceres) y actualmente

\footnotetext{
21 Vid el mapa de la distribución de los Iulii en la Península Ibérica en Guichard, P. y LEFebvre, S.: "Un Nouveau vicus en Hispania d'après une inscription inédite d'Estrémadure". Melanges de la Casa de Velázquez, XXVIII, I, 1992, p. 169.

${ }_{22}$ Vid. CIL II 454; HEp 2, 1990, 770 y, sobre todo, el trabajo reciente de ABASCAL PALAZÓN, J. M., titulado: Los nombres personales en las inscripciones latinas de Hispania. Murcia, $1994, p$.

23 CIL $\| 146$.

24 A. E. $1984,468$.

25 CPILC, 464.

26 Vid. sobre este nombre: Albertós Fimat, M. L.: La Onomástica, op. cit., pp. 21-22 y Palomar Lapesa, M.: La Onomástica Personal Pre-latina de la Antigua Lusitania. Salamanca, 1957, pp. 33-44; UntERMANN, J.: Elementos de un atlas antroponímico de la Hispania Antigua. Madrid, 1965, pp. 53-54, mapa nำ 7 . Y el trabajo ya reseñado de Abascal PALAzón, J. M.: Los nombres, op. cit., p. 272.
} 272. 
está desaparecida. La lectura e interpretación que hizo del editor del neto inscrito fue la siguiente ${ }^{27}$ :

$$
\begin{aligned}
& \text { L(ucius) } \cdot \text { CAV(ius) S(exti) } \\
& {[(\text { (Flius) B]ALANVS }} \\
& \text { Lac(ui) } \cdot V \text { (otum) } \cdot S \text { (olvit) }
\end{aligned}
$$

Con la tipografía con la que entonces se contaba, Roso de Luna hizo notar en el texto de la inscripción la falta del primer trazo vertical en la «B $B$ y el nexo "AL " de la Línea 2. Este autor completó el texto del epígrafe y apuntó que el nombre abreviado de la divinidad a la que se había consagrado el ara correspondía a $L a c(u i)$, es decir, a un supuesto dios lago, apostillando que "Los lagos, así como los ríos y montes, se adoraban» ${ }^{28}$.

A pesar de la inexistencia de paralelos en la epigrafía, la interpretación de $L a c(u i)$ fue aceptada por casi todos los investigadores y este nombre pasó a formar parte a partir de entonces de los repertorios de teónimos indígenas atestiguados en la Península lbérica ${ }^{29}$. Estaba claro que ni Roso de Luna ni otros epigrafístas se habían percatado del hallazgo en Mérida, poco años antes, de un ara consagrado a una divinidad lusitana llamada Lacipaea ${ }^{30}$.

El hallazgo del epígrafe inédito consagrado a Lacipaea en Conquista de la Sierra nos ha obligado a revisar la epigrafia latina de la provincia de Cáceres, permitiéndonos reinterpretar el texto epigráfico del citado ara de Torre de Santa Maria publicada por Roso de Luna hace ya bastantes años y que, a nuestro juicio, también está consagrada a la diosa lusitana.

La lectura y transcripción que nosotros ofrecemos de su texto epigráfico es la siguiente:

$$
\begin{aligned}
& \mathrm{L}(\text { ucius }) \cdot \operatorname{CAV}(-\cdots) \mathrm{S}(\text { exti) }[(\mathrm{F} \text { (ilius) } \\
& {[\text { B]ALANVS }} \\
& \mathrm{LAC}(\text { ip o b? aeae }) \cdot \mathrm{V}(\text { otum }) \cdot S(\text { olvit })
\end{aligned}
$$

\footnotetext{
27 Roso DE LUNA, M.: “Nuevas inscripciones romanas de la región norbense". BRAH, XLVII, 1905, p. 69, nº 78; CPILC 501.

${ }^{28}$ ROSO DE LUNA, M.: "Nuevas inscripciones", op. cit., p. 69.

29 En el CPILC, 501 se ofrece la siguiente lectura: L-Caus / Balanus / Lac-v-s interpretando: L(ucius) Caus / Balanus lac(ui) v(otum) s(olvit).

30 El epigrafe está actualmente desaparecido. Vid. FITA, F.: “Excursiones Epigráficas». BRAH XXV, 1894, pp. 94-95. Y HüBNER (EE VIII, 364-365, $n^{9} 23$ ). El texto del epigrafe decia lo siguiente: [Nerone] Claudio: Caesare- III- co(n)s(ule) / Vitulus et-Proculus-Valeri(i)-fratres/Tarmes(ini)Lacipaea-votum- solver(unt)-1(ibentes)-m(erito).
} 
El precedente que representa el hallazgo de varias inscripciones latinas consagradas a Lacipaea en los territorios de esta zona concreta de la Lusitania junto con la ubicación de la mansio del mismo nombre cerca de Augusta Emerita, parecen confirmar que este ara votiva pertenece a la conocida divinidad lusitana.

El grado de conocencia y familiaridad alcanzado por la divinidad Lacipaea entre las gentes que poblaron los territorios citados viene a justificar que el nombre del teónimo aparezca abreviado en el texto epigráfico.

\subsection{Fuentes textuales sobre Lacipaea}

El nombre de Lacipaea aparece documentado en los Itineraria hispano ${ }^{31}$ como primera mansio del Alio Itinere ab Emerita Caesaraugustam, es decir, de la vía de comunicación terrestre que unía a Complutum con Augusta Emerita. Este núcleo de población estaba posicionado a veinte millas romanas de Mérida, es decir, a unos treinta kilómetros aproximadamente de la capital de la Lusitania.

Desde el siglo $X I X$ se han realizado múltiples intentos para localizar el asentamiento de Lacipaea. Sin embargo, todavía hoy su localización no está resuelta de una forma definitiva y satisfactoria. Se han propuesto diversas ubicaciones para su asentamiento, destacando entre ellas: en el camino de Mérida a Trujillo ${ }^{32}$; alrededores de Madrigalejo ${ }^{33}$; "Venta de la Guía" en Santa Amalia ${ }^{34}$; y recientemente Albalá ${ }^{35}$.

En honor a la verdad, unas propuestas parecen más realistas que otras. Sin embargo, ninguna de ellas ha conseguido situar el posicionamiento exacto de este establecimiento urbano que a la vez era mansio de la via de comunicación terrestre que unía a Augusta Emerita con Complutum.

31 Itinerarium Antonini Augusti. Itinera Romana, vol., prius., edit. de Cunz, Leipzing, 1929, 438, 4 y Ravennatis Anonymi Cosmographia, Itinera Romana, vol, alterum. ed. de J. Schnetz. Leipzing, 1929, 312, 16.

32 Roso DE LUNA, M.: “Las vias romanas del Nordeste de Mérida". BRAH LX, 1912, pp. 374 y ss.

33 FITA Y COLOME, F.: «EXcursiones epigráficas", BRAH XXV, 1894, pp. 43-166.

34 MÉlIDA, J. R.: Catálogo, op. cit, p. 371.

35 Rosco Madruga, J., y TÉllez JimÉnez, M. L.: “ ¿Lacipea ubicada?" en Manifestaciones religiosas de la Lusitania, Cáceres, 1985, pp. 132-133. 


\subsection{Fuentes epigráficas}

Hace ya más de un siglo que F. Fita publicó un texto votivo inédito grabado en una placa de mármol blanco de $21 \mathrm{cms}$. de altura y $120 \mathrm{cms}$. de longitud, que se habia encontrado en Mérida, donde por primera vez se constataba el nombre de Lacipaea en un monumento epigráfico. Sabemos que la fecha de la dedicación corresponde al año $58 \mathrm{~d}$. C (tercer consulado) y que el nombre del Emperador fue borrado intencionadamente de la placa a causa de la damnatio memoriae decretada por el Senado Romano contra Nerón. El texto del epígrafe, según su editor, decia lo siguiente ${ }^{36}$.

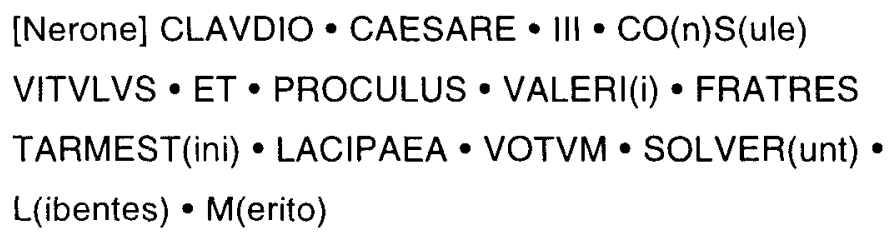

Este autor interpretó el epígrafe como una ofrenda hecha por las gentes de Lacipaea a una divinidad, quizá a Netón o a Marte, y de ahí que considererase a Lacipaea simplemente como un topónimo y nunca como una divinidad.

M. L. Albertós, al realizar una selección de los nuevos teónimos en Hispania, llegó a comentar del contenido de este epígrafe: "Lacipaea es el nombre de una ciudad próxima a Mérida y simplemente indica el lugar dónde se cumplió el voto" ${ }^{37}$. Al mismo tiempo afirmaba que el epígrafe estaba consagrado a una divinidad cuyo nombre o bien no podía pronunciarse $o$ bien era tan conocido que se sobreentendia. Esta claro, pues, que esta autora consideraba a Lacipaea como un topónimo y nunca como un teónimo.

Años después, J. M. Blázquez ${ }^{38}$ se enfrentaba de nuevo al problema y se inclinaba a considerar a Lacipaea, aún admitiendo la posibilidad planteada por Albertós, como el nombre de la diosa de la ciudad de Lacipea, posicionada como primera mansio del Alio Itinere ab Emerita Caesaraugustam.

\footnotetext{
${ }_{36}$ FITA, F.: «Excursiones Epigráficas». BRAH, XXV, 1894, pp. 94-95. Vid. también EE VIII, 364-365, $\mathrm{n}^{\circ} 23$.

37 Albertós Firmat, M. L.: "Nuevas divinidades de la Antigua Hispania». Zephyrus III, Salamanca, 1952, p. 63.

3a Blazquez Martinez, J. M.: Religiones Primitivas de Hispania. Fuentes Literarias y Epigráticas. Roma, 1962, p. 79.
} 
Pasada una década, L. Garcia Iglesias, apoyándose en el texto del epigrafe objeto de estudio, se decanta en contra de la suposición de Blázquez y propone "como alternativa la hipótesis indemostrable de que en Tarmest esté el nombre de la divinidad" ${ }^{39}$.

La consideración de Lacipaea como topónimo o como teónimo distaba de estar clara y se seguía considerando más, en general, primera posibilidad que segunda. También es verdad que la falta de paralelos seguros anteriores al hallazgo de la inscripción de Mérida en la epigrafía peninsular, no ayudaba en nada a saber si el nombre de Lacipae realmente, además de ser el topónimo de una ciudad situada a unos treinta kilómetros de Augusta Emerita, correspondía también al teónimo de la diosa de la ciudad.

En $1986 \mathrm{~J}$. Rosco y L. Téllez ${ }^{40}$ dieron a conocer el texto epigráfico de una nueva ara votiva de material granítico fragmentada en su parte inferior (Vid. Figura 5). Formaba parte de la pared exterior de la trasera de la casa no 13 de la calle Mérida, situada muy próxima al "Cruce de las Cuatro Callejas», en la localidad cacereña de Albalá.

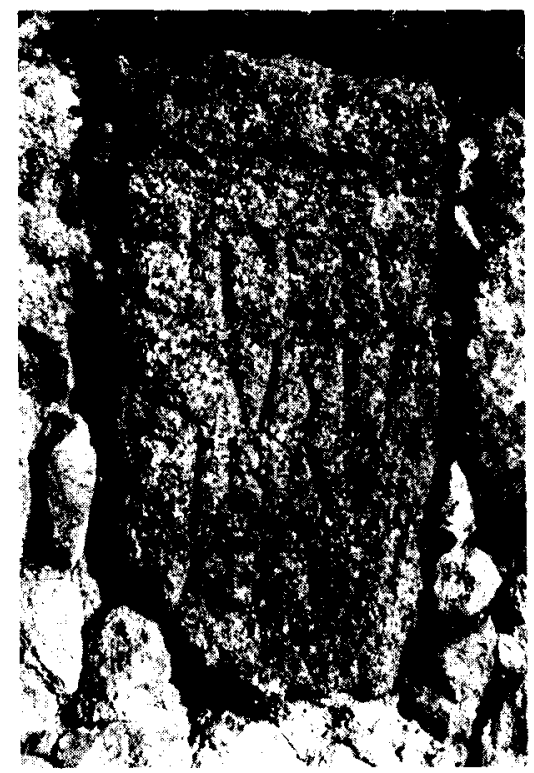

Figura 5

39 Garcia Iglesias, L.: Epigrafia romana de Augusta Emerita. (Tesis Doctoral Inédita). Madrid, 1972, p. 58.

40 Rosco Madruga, J. y TÉLLEZ JIMÉNEZ, L.: «Lacipea Ubicada?» en Manifestaciones religiosas en Lusitania, Cáceres, 1986, pp. 133-134. 
Las medidas visibles de la inscripción son de $56 \mathrm{cms}$. de altura por 30 $\mathrm{cms}$. de anchura. Su campo epigráfico es de $33 \mathrm{cms}$. de altura por $30 \mathrm{cms}$ de anchura. Las letras, cuya altura oscila entre entre 8 y $6 \mathrm{cms}$., están ligeramente desgastadas, pero no plantean problemas de lecturas. Son capitales cuadradas con rasgos rústicos.

La lectura del epígrafre ofrecida por ambos autores fue la siguiente:

\author{
GIPEIE \\ CVS LA \\ CIBAEA \\ $V \cdot S \cdot L \cdot M$
}

Inicialmente creyeron que estaba consagrada a un supuesto dios lusitano llamado Gipeicus. Era obvio que ambos editores sólo se ocuparon de considerar y argumentar el hallazgo de Lacibaea como topónimo, no planteándose la posibilidad de que fuera el nombre de la conocida divinidad lusitana atestiguada en el epígrafe de Mérida. Sin embargo, en un estudio posterior Rosco ${ }^{41}$ abre la posibilidad de que pudiera tratarse de una dedicación a la diosa Lacipea.

Afortunadamente, en un trabajo reciente Gamallo y Madruga ${ }^{42}$ presentan una nueva interpretación, a nuestro juicio acertada, del texto epigráfico de este ara y que dice lo siguiente:

\title{
G I-PELE \\ CVS LA- \\ CIBAEA
}

VS L M

Transcripción: G(aius) I(ulius?) - PELE/CVS LA/CIBAEA N(otum) S(olvit) L(ibens) M(erito).

Para Gamallo y Madruga el supuesto teónimo Gipeicus que habian interpretado inicialmente Rosco y Téllez en el texto del epígrafe corresponde

41 Caballero Zoreda, L., y Rosco Madruga, J.: «Iglesia visigoda", op. cit., p. 240.

42 Gamallo Barranco, J. L., y Madruga Flores, J. V.: "Nuevos epigrafes extremeños (sur de Cáceres y norte de Badajoz)". Gerión, 12, 1994, pp. 288-290. 
en realidad a los tria nomina del dedicante del ara, es decir, a un tal G(aius) lulius Pelecus., individuo en posesión de la ciudadanía romana, pero de clara raigambre hispana como denuncia su cognomen, novedoso en la epigrafía latina de la Península Ibérica.

A la luz de los nuevos hallazgos epigráficos, la cuestión suscitada parece ya resuelta: el término Lacipaea hace alusión tanto a un topónimo alusivo a una ciudad situada en la vía que unía a Augusta Emerita con Complutum como al teónimo de la diosa de la ciudad del mismo nombre.

\section{3. Sobre el nombre de Lacipaea}

Hasta el momento tenemos atestiguadas cuatro epígrafes consagrados Lacipaea; en tres de ellos, el teónimo aparece en inscriptio plena, en el otro está abreviado. En las inscripciones de Mérida y Albalá se atestigua el nombre de la divinidad bajo las formas Lacipaea y Lacibaea. En este último caso, una bilabial oclusiva sonora, "B», ocupa la misma posición que una bilabial oclusiva sorda, «P».

En la inscripción de Conquista de la Sierra el nombre de esta divinidad lusitana presenta una ligera variante gráfica, Laci[p]aia o Laci[b]aia, frente a las conocidas con anterioridad, Lacipaea o Lacibaea. También queremos resaltar que en este epígrafe el teónimo de la diosa lusitana aparece representado en dativo en "ae", Laci[p]aiae o Lacilb]aiae (sic), y no, como era lo habitual en las inscripciones conocidas, en "a", Lacipaea - Lacibaea. En el fondo, las ligeras variantes que presenta el nombre de la divinidad lusitana son variaciones de pronunciación del teónimo de la diosa y que se reflejan en la forma de escribirlo, tal y como ocurre con otros teónimos ${ }^{43}$.

El nombre de Lacipaea o de sus variantes Lacibaea Lacilp]aia o Laci[b]aia es hispano con evidente carácter céltico, aunque no poseemos información que nos permita conocer su significado concreto. Contamos otros nombres en la toponimia de la Península lbérica que están participados por el mismo radical. Sirvan como botones de muestras, entre otros, los más conocidos: Lacimurga, Lacini, Laquiniesi o Lacilbula.

43 Especialmente con Ataecina. Ver el cuadro 4 de CABALlero ZorEDA, L. et alii: "La Iglesia de época visigoda", op. cit., 1991, p. 509. 


\section{4. Ámbito de culto}

Hasta el momento contamos con cuatro testimonios epigráficos consagrados a la diosa lusitana Lacipaea en la Península Ibérica. Todos ellos han sido hallados en localidades extremeñas, concretamente, en Mérida, Albalá, Torre de Santa María y Conquista de la Sierra, es decir, dentro de la jurisdicción administrativa del antiguo conventus Emeritensis (Vid. el mapa).

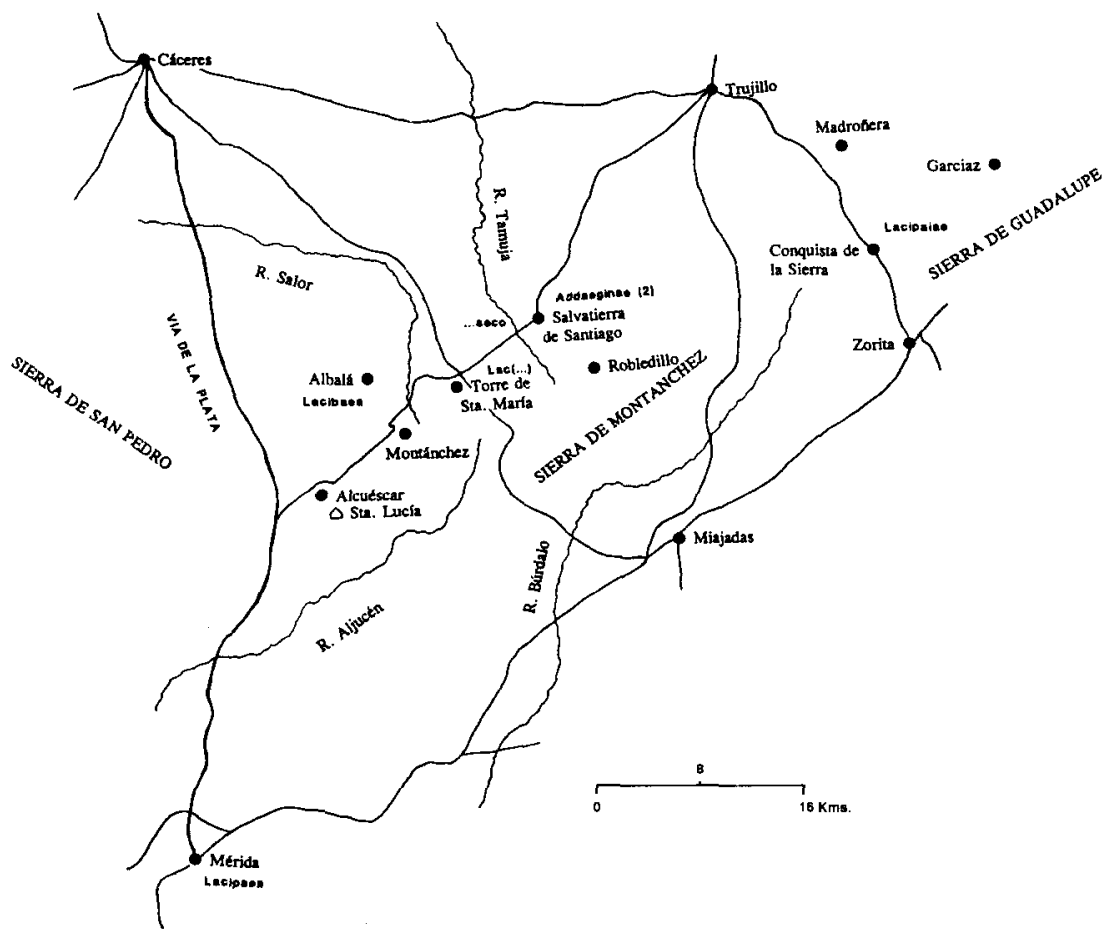

A juzgar por la distribución de los testimonios epigráficos documentados hasta el momento, el ámbito de culto de Lacipaea se reduce a una zona restringida ${ }^{44}$, cuya delimitación geográfica corresponde, a grandes rasgos, al triángulo formado por Mérida, Cáceres y Trujillo, territorios perteneciente en la Antigüedad a Augusta Emerita, Praefectura Turgalensis y Norba Caesarina.

44 Su ámbito de culto es prácticamente coincidente con el área de la distribución geográfica de las aras cacereñas a Liber-Libera. Vid. al respecto el trabajo de MELENA, J. L.: "Notas de epigrafía romana de Extremadura: I. Un pretendido teónimo nuevo en Lusitania». Velieia, 7, 1990, p. 150. 
El centro de culto de esta divinidad lusitana estaría, obviamente, en la ciudad de Lacipea, situada, segun los Itinerarios antiguos, a treinta kilómetros aproximadamente al norte de Augusta Emerita.

Lacipaea sería, quizás, una divinidad lugareña, protectora de las gentes, campos, ganados y posesiones en general, perteneciendo al grupo de dioses cuyo nombre es un topónimo.

\section{INSCRIPCIÓN INÉDITA DE ..[- - - ]AECO (Figura 6)}

Inscripción votiva inédita. Fragmento inferior de ara de granito rojizo claro, de procedencia no local, de la que se ha conservado parte del fuste y todo su zócalo. Fue encontrada en 1991 por D. Francisco. Hurtado Sáez ${ }^{45}$, en la finca denominada "El Calvillo", situada en el término municipal de Salvatierra de Santiago (Cáceres). Actualmente se encuentra depositada en el patio de la casa de su descubridor, calle José Antonio no. 21 , en la localidad de Valdefuentes.

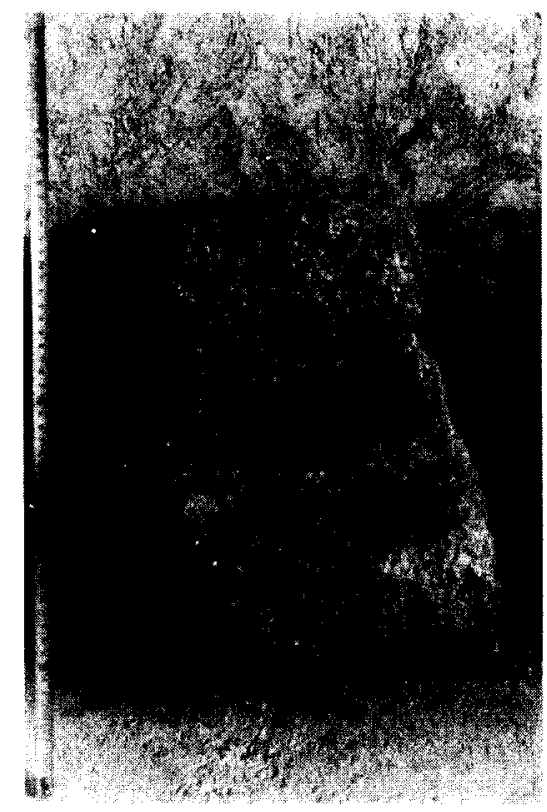

Figura 6

45 Queremos expresar nuestro más sincero agradecimiento a Don Francisco Hurtado Sáez, por su amabilidad y facilidades dadas para el estudio del ara. 
El paraje donde se exhumó el fragmento de ara pertenece a una zona donde se documentan numerosas villae rústicas.

Las dimensiones del fragmento del ara son $37 \mathrm{cms}$. de altura, por 24 $\mathrm{cms}$. de ancho, y de un grosor de $18 \mathrm{cms}$. El tipo de letra del epigrafe es capital cuadrada con ápices con rasgos rústicos, de muy buen trazo, y con alturas decrecientes en sus líneas en $0,5 \mathrm{cms}$. y que oscila de 4,5 cms. en Lín. 1, hasta 3,5 cms. en Lín. 3. Sólo en la Lín. 2 presenta un punto redondo.

El texto del epigrafe dice lo siguiente:

\author{
AECO \\ CALPVR(nius-a?) \\ CLEME(n)S
}

Transcripción: - . . . . / AECO / CALPVR(nius-a?) / CLEME(n)S

Nexos: Línea 2: A y L; $V$ y R.

El aspecto final que nos ofrece el epígrafe es globalmente agradable, circunstancia no muy frecuente en esta zona de la Lusitania, con una ordenación cuidada y una estampa, en cierto modo, elegante. Ello se debe tanto al tipo de letra y a la colocación de las palabras unidas en nexo como a la interpunción que presenta, meramente ornamental. Los ingredientes esgrimidos nos lleva a la conclusión de que el taller donde se elaboró el ara no sería, casi con seguridad, de procedencia local, por lo que hubo de encargarse y traerse o bien de la no lejana Emerita Augusta o bien de la más cercana Turgalium.

El esquema básico de las inscripciones votivas latinas consta, casi siempre, de tres elementos intercambiables, pero no sustituibles, entre sí. En primer lugar suele aparecer, en los epígrafes más antiguos, el nombre de la divinidad - aunque muchas veces si es conocida por el contexto se omite-, después el nombre del oferente $y$, por último, la fórmula ritual y/u ofrenda.

El esquema epigráfico habitual para las inscripiones votivas en esta zona de la Lusitania se desarrolla según el modelo propuesto. Nuestra inscripción es, sin duda, una dedicatoria, aunque no podemos asegurar si la parte inicial del texto epigráfico desaparecido contenía la ofrenda, si ésta era real o no (aram, ex voto,...), o si bien contenía sólo la fórmula votiva. 
El sufijo conservado del ara, -aeco, no sabemos realmente si ayuda a configurar el teónimo o simplemente participa en la formación de algún epíteto que acompaña a la divinidad. En todo caso se trata de una terminación adjetival compuesta de un sufijo de derivación, en variante sorda $-k o$, precedido de la parte final del tema - ae-, que desarrolla un diptongo decreciente al contacto con la oclusiva " $\mathrm{C}$ ".

Epítetos, con la misma terminación adjetival que la del teónimo del epigrafe, se han hallado en inscripciones localizadas en Alburquerque, Aeco ${ }^{46}$; en Coria a la pareja de divinidades Arentio/Arentio le acompaña el epíteto Amrun(aeco) ${ }^{47}$; en Brozas se encontraron varios epígrafes dedicados a Bandia Apuluseaeco ${ }^{48}$; en un ara procedente de un lugar incierto del norte de la provincia de Cáceres se atestigua, según la lectura ofrecida ${ }^{49}$, Cantunaeco; y en Coria se descubrió una inscripción consagrada al dios Eaeco ${ }^{50}$.

En la localidad cacereña de Ruanes encontramos ofrecimientos epigráficos a Revve Anabaraeco ${ }^{51}$ y a Roudaeco; este último dios también aparece atestiguado junto con Baraeco en Trujillo ${ }^{52} \mathrm{y}$, con el prefijo

46 En la ermita de Santiago, sin más datos: aeco / B Crescens / S(olvit) (HEp 1, 1989, 80).

${ }_{47}$ El texto del epigrafe dice lo siguiente: Aren/ tiae/ Arentio/ Am[r]un(aeco)/ Tanc[i]n(us) / Caturi [f(ilius)] / v(otum) s(olvit) l(ibens) m(erito) (Vid. CPILC, 772).

48 Los textos de los epígrafes consagrados a Bandía Apuluseaeco dicen lo siguiente: Cilius / Caenonis / f(ilius) Apulus/eaeco / v(otum) s(olvit) l(ibens) m(erito) (Vid. CIL II 741 y CPILC, 519 y, sobre todo, Salas Martin, J.; Esteban Ortega, J.; Rueda Muñoz, de San Pedro, G.: "Bandia Apolosecus, una divinidad con culto local en la zona de Brozas, Cáceres", H. A. 11-12, 1989, $n^{\circ} 3$ foto); y: Apulusea[e]/co Sala[ei]/us R(ufi) f(ilius) / a(ram) p(osuit) (SALAS MARTín, J.; ESTEBAN Ortega, J.; Rueda Muñoz, de San Pedro, G.: «Bandia Apolosecus», op. cit., 10, n 1 foto y HEp 3 , 1993, 119).

49 De origen incierto de la provincia de Cáceres, se conserva en Ciudad Rodrigo y en ella se lle: Silo Co/rai (filius) B/cantu/naeco / v(otum) a(nimo) I(ibens) s(olvit) (Vid. CIL II 861; BLÁzQUEZ Martinez, J. M.: Religiones Primitivas, op. cit., 1962, 208).

5o Aunque se duda de la lectura del teónimo, pudiéndose transcribir Eaco en lugar de Eaeaco, nos sigue sirviendo. Dice $\mathrm{D}(\mathrm{eco})$ Eaeco / Clara/nus $\mathrm{Ca} / \mathrm{enici}<\mathrm{f}$ (ilius) > v(otum) / s(olvit) l(ibens) m(erito) (CIL II 763. CPILC 204).

51 Dice: Revve Ana/baraeco / After Albini / f(ilius) Turolus / v(otum) s(olvit) I(ibens) m(erito) (CIL II 685 y CPILC, 422).

52 VId. BELTRÁN LLORIS, M.: (“Aportaciones a la epigrafía y arqueología romana de Cáceres". Caesaraugusta $39-40,1975-1976$, núms. 60 y 64) da a conocer dos inscripciones consagrada a Roudaeco. Aunque ya Hübner consideraba sospechosa la inscripción, sigue valiéndose el nombre del teónimo. Su texto es: Baraeco / Nobb(...) ac / lunius aed(il?) / II vir (?) C(aesaris) l(mperatoris) / pro salute (CIL II 5.276. CPILC 555. HEp 4, 1994, 253). Del siguiente teónimo tenemos: ...Roud/aeco v(otum) s(olvit) / a(nimo) l(ibens) (Vid. BELTRÁN LlorIs, M.: "Aportaciones", op. cit., pp. 88-89, $n^{\ominus} 60$ foto) y ...[Cau]/qiri f(ilius) $B / a(n)^{\wedge} d u(e) R o /[u d e] a e c / o$ a(nimo) l(ibens) / v(otum) [s(olvit) (Vid. BeltaAn LloRIS, M.: “Aportaciones", op. cit., pp. 88-89, n²61). Aún cabria aumentar el catálogo con otro epígrafe que se publicó como aparecido en Casar de Cáceres, que se conserva en una colección particular de Cáceres y que al parecer procede de la zona de Trujillo: 
Bandue, en Madroñera ${ }^{53}$. La presencia de varias inscripciones votivas consagrada a Roudaeco en la comarca de Trujillo nos invita a pensar en la posibilidad de que estemos frente a una nueva dedicación a este dios. Sólo podría asegurarse si se hallara la parte superior del ara que estamos estudiando.

Con respecto al gentilicio Calpur, al aparecer sin praenomen y terminar abreviado no podemos saber con certeza el género del dedicante. Personalmente nos inclinamos por un nomen en masculino, pues los epígrafes hallados en la zona ${ }^{54}$ y en el mismo pueblo de Salvatierra de Santiago ${ }^{55}$ asi lo aconsejan. El cognomen, en esta misma grafía, Clemes, lo encontramos otra vez en el mismo municipio ${ }^{56} \mathrm{y}$, curiosamente, allí mismo se descubrió un personaje llamado $Q$. Calpurnius Clementinus.

L(ucius) Aemi/lius Proc/ulus vica/nis Roud(ensibus?) / d(e) s(uo) f(aciendum) c(uravit) (A. SANCHEZ PAREDES: "Extremadura", 20 y 24/01/1967. HEp 2, 1990, 211). Recientemente se ha hallado una dedicación a Ceres (Cereri / L(ucius) Iulius / L(uci) f(ilius) Afer / vicanis d(edit) d(edicavit) "Extremadura" 06/05/92 foto. HEp 3, 1993, 97) cerca de Lacinimurga en el norte de la provincia de Badajoz. Si se observan ambas inscripciones se ve que existe un paralelismo casi idéntico, y por ello habria que reinterpretar la que tratamos, en el sentido siguiente: Lucio Emilio Proculo, con su dinero, se preocupó de hacer construir el monumento, en homenaje a un dios Roud(eaco), en nombre de los habitantes de su vicus.

53 Bandue / Roudeae/co a(nimo) I(ibens) p(osuit) / ...... (BeLtRÁN LlORIS, M.: "Aportaciones", op. cit., pp. 92-93, no 64, foto. AE 1977, 432.

54 En Casas de Don Antonio: lovi Opt/[imo] Max(umo / [C]alpurn/[iu]s Proti/[nu]s v(otum) s(olvit) I(ibens) m(erito) (Vid. Redondo Rooriguez, J. A., y Solana Pujalte, J.: "Aportaciones a la epigrafia latina de la Alta Extremadura", Anuario de Estudios Filológicos, 8, 1985, pp. 271-272 no 5 foto) y en Valdefuentes: Calpu/rnius / L(ucii) [l(ibertus)] Rus/tii]cus / h(ic) s(itus) e(st) s(it) / t(ibi) [t(erra) I(evis)] (Roso DE LUNA, M.: "Nuevas inscripciones", op. cit., 1904, p. 124; CPILC 580; y GonzAlez Cordero, A. et alii. "Nuevas aportaciones", op. cit., pp. 66-67 foto).

55 Tres paralelos nos encontramos: 1.- $L$ (ucius) Calpurnius $P . . /$ an(norum) $L X X X[h$ (ic) s(itus) (Vid. CIL II 996; EE IX, 1903, 62 no 996). 2.- D(is) M(anibus) S(acrum) / Q(uintus) Calpurn/iusClem/entinus / an(norum) LXX Su/ra Vitula / socero p(issimo) / $\mathrm{f}$ (aciendum) c(uravit) $\mathrm{h}$ (ic) s(itus) e(st) s(it) t(ibi) t(erra) l(evis) (Vid. MALLON, J. y MARTín, T.: Las inscripciones, pp. 210211, n² 229; CPILC 435). 3.- [.]Calp/[ur]nius / [Ânc?] teti (ilius) / [G]racil/[is] ân(norum) LXV / [h(ic) s(itus) e(st)] s(it) t(ibi) t(erra) l(evis) (Inédita, recientemente descubierta por M. Morales Moreno).

56 Lectura de $M$. Roso y corregida la filiación por J. RAMON Y FEANÁNDEZ-OXEA: Q(uintus) Norba/nus Clem/es Macaldici f(ilius) h(ic) s(itus) e(st) (Vid. RAMÓN y FERnÁNDEZ-OXEA, J.: “De epigrafia", op. cit., p. $192 \mathrm{n}^{\circ} 24$ foto. y CPILC 431). 